\title{
Incoherent Control of Locally Controllable Quantum Systems
}

\author{
Daoyi $\operatorname{Dong}^{1,2 *}$ \\ 1. Institute of Cyber-Systems and Control, \\ National Laboratory of Industrial Control Technology, \\ Zhejiang University, \\ Hangzhou 310027, China \\ 2. Institute of Systems Science, \\ AMSS, Chinese Academy of Sciences, \\ Beijing 100190, China \\ Chenbin Zhang \\ Department of Automation, \\ University of Science and Technology of China, \\ Hefei 230027, China \\ Herschel Rabitz and Alexander Pechen \\ Department of Chemistry, \\ Princeton University, Princeton, \\ New Jersey 08544 USA \\ Tzyh-Jong Tarn \\ Department of Electrical and Systems Engineering, \\ Washington University in St. Louis, \\ St. Louis, MO 63130 USA
}

(Dated: November 12, 2018) 


\begin{abstract}
An incoherent control scheme for state control of locally controllable quantum systems is proposed. This scheme includes three steps: (1) amplitude amplification of the initial state by a suitable unitary transformation, (2) projective measurement on the amplified state, and (3) final optimization by a unitary controlled transformation. The first step increases the amplitudes of some desired eigenstates and the corresponding probability of observing these eigenstates, the second step projects, with high probability, the amplified state into a desired eigenstate, and the last step steers this eigenstate into the target state. Within this scheme, two control algorithms are presented for two classes of quantum systems. As an example, the incoherent control scheme is applied to the control of a hydrogen atom by an external field. The results support the suggestion that projective measurements can serve as an effective control and local controllability information can be used to design control laws for quantum systems. Thus, this scheme establishes a subtle connection between control design and controllability analysis of quantum systems and provides an effective engineering approach for controlling quantum systems with partial controllability information.
\end{abstract}

PACS numbers: 42.50.Dv, 02.30.Yy, 03.65.-w

*Electronic address: dydong@amss.ac.cn 


\section{INTRODUCTION}

Control of quantum phenomena plays an important role in various research fields including physical chemistry [1, 2, 3, 4, 5], atomic and molecular physics [6, 7, 8], quantum information [9, 10, 11] and future quantum technologies [12]. In quantum control theory, the controllability of quantum systems is the first fundamental issue to address [13] due to its practical importance, including a close connection with the universality of quantum computation [14], the possibility of attaining atomic or molecular scale transformations [15, 16], etc. Different notions of controllability exist including pure state controllability, complete controllability, wavefunction controllability, and kinematic controllability in the set of all density matrices [15]-[22]. A common research focus is on finite dimensional quantum systems for which the controllability criteria may be expressed in terms of the structure and rank of the corresponding Lie groups and Lie algebras [2]. This method allows for the easy mathematical treatment of closed quantum systems and in some cases can directly benefit from classical control theory. Various results have been derived for specific problems using this method [17, 18], but the relevant criteria may be computationally difficult when the dimension of the controlled system is large. Turinici and Rabitz [19, 20] proposed a wavefunction controllability method based on graph theory, and its controllability criterion becomes easy to verify.

Most of the existing results consider control within the whole state space of controlled quantum systems, and we call this global controllability. In practical applications, some quantum systems may not be globally controllable, or the information about global controllability may be difficult to acquire. However, it may be easy to obtain local controllability information. For example, Beauchard [23] proved that a nonrelativistic charged particle in a 1-D box controlled by a uniform electric field is locally controllable around the ground state. Furthermore, Beauchard and Coron [24] also proved that in some cases two eigenstate locally controllable wavefunctions can be moved exactly from one to another in a finite time. Local controllability has also been an interesting topic for classical mechanical systems [25] and in chemical reaction control [26]. Moreover, in some situations, the focus may only be on the control within some state subspace of the controlled system. For example, in quantum computation one may only focus on decoherence-free subspaces (DFS) [27, 28, 29]. The control design of locally controllable quantum systems is very relevant for such problems 
and in this paper we focus on quantum systems with local controllability information.

The effective determination of control strategies is another important problem in quantum control theory. The main paradigm is coherent control where one manipulates the state of the system by applying a semiclassical potential in a fashion that preserves quantum coherence [30]-[34]. This approach has successfully been used, e.g., for control of chemical reactions via two-beam interference control and two-pulse time delay control [33, 34]. The method of learning control has been developed [6], which has the essential advantage of being applicable to control problems lacking detailed information about the controlled system and its dynamics.

In coherent control, quantum measurements are commonly viewed as having a deleterious effect, since they destroy the coherent state of the measured system. However, recent results show that in some situations quantum measurements can be beneficial for quantum control, and they can be combined with unitary operators to achieve certain quantum control tasks or even can make nonunitarily controllable systems controllable [35]-[41]. In these schemes quantum measurements destroy the coherent characteristics of the controlled systems, and thus such control can be called "incoherent control". Various methods for direct incoherent control by the environment were also proposed [42, 43, 44, 45].

In this paper, we propose an incoherent control scheme for locally controllable quantum systems. This scheme can be considered as a specific type of measurement-assisted control [39, 41] and includes three steps: (1) amplitude amplification of the initial state, (2) projective measurement on the amplified state, and (3) subsequent transfer of the resultant state into the target state. The first and the last steps are realized by unitary operators, and thus correspond to coherent control. The intermediate step is realized by a measurement, and thus corresponds to incoherent control. The first step enhances the probability of success by enhancing the amplitude of some desired system eigenstates. The second step projects, with high probability (the probability of success), the amplified state onto a desired eigenstate. The last step transfers the desired eigenstate into the target state. This quantum control method is probabilistic due to the nature of quantum measurements; however, the probability of success can be greatly enhanced by amplitude amplification technology [46, 47, 48]. Within this scheme, we present two incoherent control algorithms: one for a target state which is reachable from a specific eigenstate, and the other for quantum systems with wavefunction controllable subspaces. As an example, we investigate the control 
of a hydrogen atom subject to an external field. The results demonstrate that projective measurement and local controllability information can be very helpful for the control design of quantum systems.

The paper is organized as follows. Section II introduces the notions of local controllability and a wavefunction controllable subspace. In Section III, the two algorithms based on the incoherent control scheme with quantum amplitude amplification and projective measurement are formulated for two classes of quantum systems. An illustration is given to demonstrate the incoherent control scheme in Section IV. Concluding remarks are given in Section V.

\section{LOCAL CONTROLLABILITY AND A WAVEFUNCTION CONTROLLABLE SUBSPACE}

This section first describes the control model for general finite-level quantum systems. Then we give the definitions of local and global controllability using the notion of a reachable set. Finally, we introduce the concepts of a wavefunction controllable subspace and wavefunction controllability, considering quantum systems with wavefunction controllable subspaces as a special class of locally controllable systems.

\section{A. Quantum Control Model}

In quantum mechanics, the state of a closed quantum system at time $t$ can be represented by a vector $|\psi(t)\rangle$ in some Hilbert space $\mathcal{H}$. The state $|\psi(t)\rangle$ of a closed quantum system evolves according to the Schrödinger equation [20]

$$
\iota \hbar \frac{\partial}{\partial t}|\psi(t)\rangle=H_{0}|\psi(t)\rangle, \quad|\psi(t=0)\rangle=\left|\psi_{0}\right\rangle
$$

where $\iota=\sqrt{-1}, H_{0}$ is the internal Hamiltonian of the system (i.e., a Hermitian operator in $\mathcal{H}), \hbar$ is Planck's constant, and the initial state has unit norm $\left\|\psi_{0}\right\|^{2} \equiv\left\langle\psi_{0} \mid \psi_{0}\right\rangle=1$. The control of the system is realized by a control function $u(t) \in L^{2}(\mathbf{R})$ coupled to the system via a time-independent Hermitian interaction Hamiltonian $H_{I}$ (e.g., dipole moment coupling). The total Hamiltonian $H=H_{0}+u(t) H_{I}$ determines the controlled evolution

$$
\iota \hbar \frac{\partial}{\partial t}\left|\psi_{u}(t)\right\rangle=\left[H_{0}+u(t) H_{I}\right]\left|\psi_{u}(t)\right\rangle
$$


The goal of the control is to find a final time $T>0$ and a finite energy input $u(t) \in$ $L^{2}([0, T], \mathbf{R})$ which drives the system from the initial state $\left|\psi_{0}\right\rangle$ into some predefined target state $\left|\psi_{\text {target }}\right\rangle$ (e.g., population transfer using external control fields).

An important problem to assess is the controllability of the system which establishes which states can be connected by the admissible controls. The controllability analysis for infinite dimensional quantum systems is typically difficult to assess. For simplification, we consider finite dimensional quantum systems, which is an appropriate approximation in many practical situations. For a finite dimensional quantum system, $\mathcal{H}$ is a finite dimensional Hilbert space, the free Hamiltonian $H_{0}$ is a Hermitian operator in $\mathcal{H}$ and its set of eigenstates $D=\left\{\left|\phi_{i}\right\rangle ; i=1, \ldots, N\right\}$ forms a basis in $\mathcal{H}$. The evolving state $\left|\psi_{u}(t)\right\rangle$ can be expanded in this basis as

$$
\left|\psi_{u}(t)\right\rangle=\sum_{i=1}^{N} c_{i}(t)\left|\phi_{i}\right\rangle .
$$

The total Hamiltonian $H=H_{0}+u(t) H_{I}$ defines a unitary evolution operator (propagator) $U\left(u, t_{1} \rightarrow t_{2}\right)$ such that for any state $\left|\psi_{1}\right\rangle$ the state $U\left(u, t_{1} \rightarrow t_{2}\right)\left|\psi_{1}\right\rangle$ is the solution at time $t=t_{2}$ of equation (2) with the initial state $\left|\psi_{1}\right\rangle$ at time $t=t_{1}$. In particular,

$$
\left|\psi_{u}(t)\right\rangle=U(u, 0 \rightarrow t)\left|\psi_{0}\right\rangle
$$

Unitarity of the propagator $U(u, 0 \rightarrow t)$ implies that the state $\left|\psi_{u}(t)\right\rangle$ evolves on the complex

unit sphere $S_{\mathbf{C}}^{N-1}:=\{|\psi\rangle \in \mathcal{H}:\|\psi\|=1\}$. Substitution of Eq. (3) into Eq. (2) gives the following equation for the coefficients $C(t)=\left\{c_{i}(t)\right\}_{i=1}^{N}[20]$ :

$$
\begin{aligned}
\iota \hbar \frac{\partial C(t)}{\partial t} & =[A+u(t) B] C(t), \quad C(t=0)=C_{0}, \\
C_{0} & =\left(c_{0 i}\right)_{i=1}^{N}, \quad c_{0 i}=\left\langle\phi_{i} \mid \psi_{0}\right\rangle, \quad \sum_{i=1}^{N}\left|c_{0 i}\right|^{2}=1
\end{aligned}
$$

Here $A$ and $B$ are the matrices of the operators $H_{0}$ and $H_{I}$, respectively, in the basis $\left|\phi_{i}\right\rangle$. The $A$ matrix is diagonal and the $B$ matrix is Hermitian [19], and in order to avoid the trivial control problem, we assume $[A, B]:=A B-B A \neq 0$.

\section{B. Local and global controllability}

In the development of quantum control theory, the controllability of quantum systems has been analyzed from different perspectives. Huang et al. [13] studied the controllability of quantum systems using Nelson's analytic domain theory. Ramakrishna et al. [15] 
investigated controllability and proposed a simple algorithm to detect their controllability. Schirmer et al. [18] obtained a sufficient condition for complete controllability of $N$-level quantum systems subject to a particular control pulse. Turinici and Rabitz [19, 20] investigated the controllability of quantum systems using graph theory and proved exact wavefunction controllability of finite dimensional models under very natural hypotheses. Albertini and D'Alessandro [17] defined several different notions of controllability for multilevel quantum systems and established some connections among these different notions. Altafini [21] studied the controllability properties for finite dimensional quantum Markovian master equations. Wu et al. [16] considered the smooth controllability of infinite-dimensional quantum-mechanical systems. Wu et al. [22] studied controllability of open quantum system subject to arbitrary Kraus-type dynamics and showed that such systems are completely density matrix controllable.

Most existing results consider controllability in the whole state space of the quantum system, i.e., global controllability, such as complete controllability [18], pure state controllability [17], and wavefunction controllability [20]. This paper considers the control problem for a class of quantum systems with local controllability information. First, we will define local and global controllability using the concept of reachable sets. We say that a state $\left|\psi_{2}\right\rangle$ is reachable from $\left|\psi_{1}\right\rangle$ at time $0<T<\infty$ if there exists a finite energy control $u(t) \in L^{2}([0, T] ; \mathbf{R})$ such that $U(u, 0 \rightarrow T)\left|\psi_{1}\right\rangle=\left|\psi_{2}\right\rangle$. The reachable set $R(|\psi\rangle)$ of a state $|\psi\rangle$ is the set of all states reachable from $|\psi\rangle$. If the reachable set covers the whole state space $S_{\mathbf{C}}^{N-1}$, the corresponding circumstance entails global controllability, that is to say, a quantum system is globally controllable if all its states are controllable in the whole state space $S_{\mathbf{C}}^{N-1}$ and therefore $R(|\psi\rangle)=S_{\mathbf{C}}^{N-1}$ for any $|\psi\rangle \in S_{\mathbf{C}}^{N-1}$. If $\left|\psi_{i}\right\rangle$ is an eigenstate $\left|\phi_{g}\right\rangle$ of $H_{0}$ and $\left|\psi_{\text {target }}\right\rangle \in R\left(\left|\psi_{i}\right\rangle\right)$, we say that $\left|\psi_{\text {target }}\right\rangle$ is eigenstate reachable. This situation with eigenstate reachable target states corresponds to local controllability and will be considered in this paper. In order to avoid a triviality, we assume $\left|\psi_{\text {target }}\right\rangle \neq\left|\phi_{g}\right\rangle$. As discussed in the Introduction, the assumption that some target states are eigenstate reachable is reasonable for various practical applications. Before presenting our incoherent control strategy for locally controllable systems, we will introduce a special class of locally controllable systems which have a wavefunction controllable subspace. 


\section{Wavefunction controllability and the wavefunction controllable subspace}

To study the controllability of the system with the evolution equation (5), Turinici and Rabitz [19, 20] associate to the system a non-oriented connectivity graph $G=(V, E)$, where the set $V$ of vertices consists of the eigenstates $\left|\phi_{i}\right\rangle$ and the set of edges $E$ consists of all pairs of eigenstates directly coupled by the matrix $B$,

$$
\begin{aligned}
G(V, E): \quad V & =\left\{\left|\phi_{1}\right\rangle, \ldots,\left|\phi_{N}\right\rangle\right\}, \\
& E=\left\{\left(\left|\phi_{i}\right\rangle,\left|\phi_{j}\right\rangle\right) ; i<j, B_{i j} \neq 0\right\} .
\end{aligned}
$$

Let $G_{k}=\left(V^{(k)}, E^{(k)}\right), k=1, \ldots, K$ be connected components of this graph. Denote by $\lambda_{i}(i=1, \ldots, N)$ the eigenvalues of the matrix $A$ and let $\nu_{i j}=\lambda_{i}-\lambda_{j}(i, j=1, \ldots, N)$. The following lemma provides the criteria for wavefunction controllability in terms of the connectivity graph [19, 20]:

Lemma 1 (wavefunction controllability) The system (5) is wavefunction controllable if the following assumptions hold:

(I) The graph $G$ is connected, i.e. $K=1$.

(II) The graph $G$ does not have "degenerate transitions", that is for all $(i, j) \neq(a, b), i \neq j$, $a \neq b$ such that $B_{i j} \neq 0, B_{a b} \neq 0: \nu_{i j} \neq \nu_{a b}$.

(III) For each $i, j, a, b=1, \ldots, N$ such that $\nu_{i j} \neq 0$ the number $\left(\nu_{a b} / \nu_{i j}\right)$ is rational.

The proof of Lemma 1 can be found in [20]. The three assumptions in Lemma 1 provide a sufficient but not a necessary condition for wavefunction controllability. In some circumstances, the assumptions (II) and (III) can be slightly relaxed [19].

For some practical systems, the assumption (I) of Lemma 1 may not be satisfied or verifying this assumption may be difficult. In such cases considering the problem of control inside an appropriate state subspace of the quantum system may be more practical. Moreover, for some controlled quantum systems partial controllability information can be relatively easy to obtain from some physical and chemical experiments. Assuming $K \neq 1$, let $G_{\omega}$ be a fixed connected component of $G(V, E)$ with the set of vertices denoted as $D_{\omega}=\left\{\left|\phi_{1}^{\prime}\right\rangle, \ldots,\left|\phi_{M}^{\prime}\right\rangle\right\}$ $(1<M<N)$. Obviously, $G_{\omega} \subset G(V, E)$ and $D_{\omega} \subset D$. Denoting the subspace generated by $D_{\omega}$ as $\Omega$, we have the following theorem [49]: 
Theorem 2 (wavefunction controllable subspace) The subspace $\Omega$ is wavefunction controllable if the following assumptions hold:

(I) The graph $G_{\omega}$ does not have "degenerate transitions", that is for all $(k, l) \neq(\alpha, \beta), k \neq l$, $\alpha \neq \beta$ such that $B_{k l} \neq 0, B_{\alpha \beta} \neq 0: \nu_{k l} \neq \nu_{\alpha \beta}$.

(II) For each $k, l, \alpha, \beta=1, \ldots, M$ such that $\nu_{k l} \neq 0$ the number $\left(\nu_{\alpha \beta} / \nu_{k l}\right)$ is rational.

Proof. Since $G_{\omega}$ is a connected component of $G(V, E)$, it is a connected graph itself. The subspace $\Omega$ is generated by the set $D_{\omega}$ of all vertices of $G_{\omega}$ and therefore Lemma 1, under the assumptions (I) and (II), immediately implies that $\Omega$ is a wavefunction controllable subspace.

As mentioned above, the property of wavefunction controllability of the system can be viewed as a type of global controllability, whereas the existence of a wavefunction controllable subspace can be considered as a form of local controllability. That is, the states in the wavefunction controllable subspace are locally controllable. In the following sections, besides the case when the target state is reachable from a specific eigenstate, we will also investigate the case when the target state belongs to some wavefunction controllable subspace.

\section{INCOHERENT CONTROL OF LOCALLY CONTROLLABLE QUANTUM SYSTEMS}

This section describes the proposed incoherent quantum control scheme. First, we briefly discuss general coherent and incoherent quantum control methods. Then we present quantum amplitude amplification technology which plays an important role in the proposed incoherent control scheme. Finally we formulate two algorithms for the incoherent control scheme: one is designed for general eigenstate reachable target states and the other for quantum systems with a wavefunction controllable subspace as discussed in Sec. IIC,

\section{A. Incoherent control}

The determination of suitable control strategies is an important objective in quantum control theory. Coherent control is a particularly powerful quantum control strategy, where the system is controlled by applying a semiclassical potential [30]. The controls commonly appear as the tunable parameters in the Hamiltonian of the system, which can directly 
affect the coherent part of the system's dynamics. The early paradigms of quantum control mainly concentrated on the open loop coherent control strategy [6, 32] which has achieved wide success, e.g., in control of chemical reactions [33]. Learning based coherent control [6] is proving to be extremely useful for manipulating quantum systems even when detailed information about their structure, coupling to the control field, etc. is not available. In recent years coherent control has also been proposed for incorporation into quantum feedback control, where measurements on the system are used to determine its state and then the measured outcome is exploited to generate a semiclassical potential applied to the system to coherently guide it into a desired state [30].

Quantum measurements generally destroy the coherent characteristics of the quantum system, and without management that circumstance can create difficulties for attaining successful control. However, in some circumstances quantum measurements can be effectively used for controlling quantum systems [35, 36, 37, 38, 39, 40, 41]. For example, Vilela Mendes and Man'ko [35] showed that under suitable conditions a system that is not controllable under unitary transformations can become controllable under the joint action of projective measurements and unitary evolution. Gong and Rice [37] found that measurements can assist a coherent strategy to control the population transfer branching ratio between degenerate product states. Sugawara [38] studied quantum dynamics driven by continuous laser fields under the measurement process and explored the possibility of measurement-assisted quantum control. Mandilara and Clark [36] proposed a probabilistic quantum control scheme via indirect measurement. Roa et al. [40] applied sequential measurements of two noncommuting observables to drive an unknown mixed quantum state to a known pure state without the use of unitary transformations. Rabitz and co-workers explored the use of nonselective von Neumann measurements in conjunction with optimal control to enhance the capability of controlling quantum systems 39, 41]. Incoherent control by manipulating the environment was also considered [42, 43, 44, 45]. Pechen and Rabitz [42, 43] presented a general method of incoherent control by the environment, where tailored incoherent radiation or a medium (e.g., atomic or molecular gas, solvent, etc.) is used as a control tool to drive the system to a target state via non-unitary evolution. Romano and D'Alessandro [44, 45] considered an incoherent control scheme where optimization of the state of an ancillary finite-level system is used to drive the controlled system to an arbitrary target state. We refer to all of these schemes as "incoherent control", since the coherent characteristics of the controlled system 
are destroyed in the process. Along this line, we propose an incoherent control scheme for a class of quantum systems where the target state is eigenstate reachable. This scheme includes three basic steps. In the first step quantum amplitude amplification of the initial state is used to increase the probability of success. In the second step a measurement is used to project, with high probability, the amplified state into a desired eigenstate. Finally, a controlled unitary transformation is used to steer this eigenstate into the target state. We will first introduce the quantum amplitude amplification concept and then describe the incoherent control algorithms.

\section{B. Quantum amplitude amplification}

Quantum amplitude amplification is a powerful ingredient in quantum algorithms [46, 47]. It is a natural generalization of Grover's quantum search algorithm, which allows for a speedup of many classical algorithms [46]-[48]. Amplitude amplification was first used by Brassard and Høyer [50] to construct an exact quantum polynomial-time algorithm for solving Simon's problem. The central task of quantum amplitude amplification is to find a suitable operator $\mathbf{Q}$ whose iterative action on the initial state can increase the probability of success roughly by a constant at each iteration, in analogy to the iteration process in probabilistic algorithms [46].

Let $|\Phi\rangle$ be a pure state of an $N$-level quantum system. This state can be represented as a superposition of some orthonormal basis states $\mathbb{X}=\{|0\rangle, \ldots,|x\rangle, \ldots,|N-1\rangle\}$ in the $N$-dimensional system Hilbert space $\mathcal{H}$, i.e., $|\Phi\rangle=\sum_{x=0}^{N-1} c_{x}|x\rangle$, where $\sum_{x=0}^{N-1}\left|c_{x}\right|^{2}=1$. A Boolean function $\chi: \mathbb{X} \rightarrow\{0,1\}$ induces two orthogonal subspaces of $\mathcal{H}$ : the "good" subspace and the "bad" subspace. The good subspace is spanned by the set of basis states $|x\rangle \in \mathbb{X}$ satisfying $\chi(x)=1$ and the bad subspace is its orthogonal complement in $\mathcal{H}$. We denote by $P_{g}$ the projector onto the "good" subspace. Every pure state $|\Phi\rangle$ in $\mathcal{H}$ can be decomposed as $|\Phi\rangle=\left|\Phi_{g}\right\rangle+\left|\Phi_{b}\right\rangle$, where $\left|\Phi_{g}\right\rangle=P_{g}|\Phi\rangle$ denotes the projection of $|\Phi\rangle$ onto the good subspace and $\left|\Phi_{b}\right\rangle=\left(\mathbb{I}-P_{g}\right)|\Phi\rangle$ denotes the projection of $|\Phi\rangle$ onto the bad subspace (here $\mathbb{I}$ is the identity operator). According to quantum measurement theory, the occurrence probabilities of the "good" state $|x\rangle[\chi(x)=1]$ and the "bad" state $|x\rangle[\chi(x)=0]$ upon measuring $|\Phi\rangle$ are $g=\left\langle\Phi_{g} \mid \Phi_{g}\right\rangle$ and $b=\left\langle\Phi_{b} \mid \Phi_{b}\right\rangle=1-g$, respectively.

Let $\mathcal{U}$ be a quantum algorithm that acts in $\mathcal{H}$ without measurements (i.e., $\mathcal{U}$ is a unitary 
operator) and let $|\Phi\rangle=\mathcal{U}|0\rangle$. Given two angles $0 \leq \varphi_{1}, \varphi_{2} \leq \pi$, a general quantum amplitude amplification can be realized by the following operator [46]

$$
\mathbf{Q}=\mathbf{Q}\left(\mathcal{U}, \chi, \varphi_{1}, \varphi_{2}\right)=-\mathcal{U} \mathcal{P}_{0}^{\varphi_{1}} \mathcal{U}^{-1} \mathcal{P}_{\chi}^{\varphi_{2}}
$$

The operators $\mathcal{P}_{0}^{\varphi_{1}}$ and $\mathcal{P}_{\chi}^{\varphi_{2}}$ conditionally change the phase of the amplitudes of state $|0\rangle$ and the good states respectively [46]:

$$
\begin{aligned}
& \mathcal{P}_{0}^{\varphi_{1}}|x\rangle= \begin{cases}e^{\iota \varphi_{1}}|x\rangle, & \text { if } x=0 ; \\
|x\rangle, & \text { if } x \neq 0 .\end{cases} \\
& \mathcal{P}_{\chi}^{\varphi_{2}}|x\rangle= \begin{cases}e^{\iota \varphi_{2}}|x\rangle, & \text { if } \chi(x)=1 ; \\
|x\rangle, & \text { if } \chi(x)=0 .\end{cases}
\end{aligned}
$$

The operators $\mathcal{P}_{0}^{\varphi_{1}}$ and $\mathcal{P}_{\chi}^{\varphi_{2}}$ can be expressed as:

$$
\begin{aligned}
& \mathcal{P}_{0}^{\varphi_{1}}=\mathbb{I}-\left(1-e^{\iota \varphi_{1}}\right)|0\rangle\langle 0| \\
& \mathcal{P}_{\chi}^{\varphi_{2}}=\mathbb{I}-\left(1-e^{\iota \varphi_{2}}\right) \sum_{\chi(x)=1}|x\rangle\langle x| .
\end{aligned}
$$

The physical implementation of $\mathbf{Q}$ can be accomplished by realizing the operators $\mathcal{U}, \mathcal{P}_{0}^{\varphi_{1}}$ and $\mathcal{P}_{\chi}^{\varphi_{2}}$ using external fields (or quantum gates). The action of $\mathbf{Q}$ can be described by the following lemma [46]:

Lemma 3 Let $\mathcal{U}|0\rangle=|\Phi\rangle=\left|\Phi_{g}\right\rangle+\left|\Phi_{b}\right\rangle$ and $g=\left\langle\Phi_{g} \mid \Phi_{g}\right\rangle$. Then

$$
\begin{aligned}
& \mathbf{Q}\left|\Phi_{g}\right\rangle=e^{\iota \varphi_{2}}\left(\left(1-e^{\iota \varphi_{1}}\right) g-1\right)\left|\Phi_{g}\right\rangle+e^{\iota \varphi_{2}}\left(1-e^{\iota \varphi_{1}}\right) g\left|\Phi_{b}\right\rangle \\
& \mathbf{Q}\left|\Phi_{b}\right\rangle=\left(1-e^{\iota \varphi_{1}}\right)(1-g)\left|\Phi_{g}\right\rangle-\left(\left(1-e^{\iota \varphi_{1}}\right) g+e^{\iota \varphi_{1}}\right)\left|\Phi_{b}\right\rangle .
\end{aligned}
$$

From Lemma 3, we can easily get

$$
\begin{aligned}
\mathbf{Q}|\Phi\rangle & =\mathbf{Q}\left(\left|\Phi_{g}\right\rangle+\left|\Phi_{b}\right\rangle\right) \\
& =\left[\left(1-e^{\iota \varphi_{1}}\right)\left(1-g+g e^{\iota \varphi_{2}}\right)-e^{\iota \varphi_{2}}\right]\left|\Phi_{g}\right\rangle+\left[g\left(1-e^{\iota \varphi_{1}}\right)\left(e^{\iota \varphi_{2}}-1\right)-e^{\iota \varphi_{1}}\right]\left|\Phi_{b}\right\rangle .
\end{aligned}
$$

Thus, we can amplify (or shrink) the amplitude of $\left|\Phi_{g}\right\rangle$ (or $\left.\left|\Phi_{b}\right\rangle\right)$ by a suitable selection of the parameters $\varphi_{1}, \varphi_{2}$ in $\mathbf{Q}$. To make this point clearer, consider the special case $\varphi_{1}=\varphi_{2}=\pi$. For the iteration process with $\mathbf{Q}(\mathcal{U}, \chi, \pi, \pi)$, we have the following theorem [46]: 
Theorem 4 (Amplitude Amplification) Let $\mathcal{U}|\mathbf{0}\rangle=|\Phi\rangle=\left|\Phi_{g}\right\rangle+\left|\Phi_{b}\right\rangle$, and $\mathbf{Q}=$ $\mathbf{Q}(\mathcal{U}, \chi, \pi, \pi)$. Then, for any $L \geq 0$,

$$
\mathbf{Q}^{L} \mathcal{U}|\mathbf{0}\rangle=\frac{1}{\sqrt{g}} \sin ((2 L+1) \theta)\left|\Phi_{g}\right\rangle+\frac{1}{\sqrt{b}} \cos ((2 L+1) \theta)\left|\Phi_{b}\right\rangle
$$

where $b=1-g, \theta$ is defined so that $\sin ^{2} \theta=g$ and $0 \leq \theta \leq \pi / 2$.

Theorem 4provides a method for boosting the initial success probability $g=\sin ^{2} \theta$. Applying the operation $\mathbf{Q}$ to the system's initial state $L$ times, the success probability becomes $g^{\prime}=$ $\sin ^{2}((2 L+1) \theta)$ and can be enhanced by choosing an integer $L$ such that $\sin ^{2}((2 L+1) \theta)$ is as close to 1 as possible.

Quantum amplitude amplification has led to a quadratic speedup for some quantum algorithms [46]. In this paper we use it as an important component for incoherent control design of quantum systems.

\section{Control algorithms}

Here we present the detailed algorithms for the proposed incoherent control scheme. First, we assume that the target state is reachable from some eigenstate $\left|\phi_{g}\right\rangle$, i.e., $\left|\psi_{\text {target }}\right\rangle \in$ $R\left(\left|\phi_{g}\right\rangle\right)$, and consider an arbitrary initial state $\left|\psi_{0}\right\rangle$

$$
\left|\psi_{0}\right\rangle=\sum_{i=1}^{N} c_{0 i}\left|\phi_{i}\right\rangle
$$

We take $\left|\phi_{g}\right\rangle$ as the good state and all $\left|\phi_{i}\right\rangle$ with $i \neq g$ as bad states, i.e., $\left|\phi_{g}\right\rangle$ corresponds to $\chi=1$ and $\left|\phi_{i}\right\rangle$ with $i \neq g$ corresponds to $\chi=0$. For a given initial state $\left|\psi_{0}\right\rangle$ we can construct an operator $\mathbf{Q}\left(\mathcal{U}, \chi, \varphi_{1}, \varphi_{2}\right)$ to enhance the amplitude of getting $\left|\phi_{g}\right\rangle$, as described in Sec. IIIB. Here $\mathcal{U}$ is determined by the initial state $\left|\psi_{0}\right\rangle$. Moreover, we need to find an optimal number of iterations $L$ to maximize the probability of success. Then we make a measurement on the enhanced conditional state such that after the measurement the system will collapse into $\left|\phi_{g}\right\rangle$ with a high probability. Finally we select a suitable control to drive $\left|\phi_{g}\right\rangle$ into $\left|\psi_{\text {target }}\right\rangle$. The main steps of the algorithm are presented in Algorithm 1 and its operation is shown in Fig. 1. The algorithm is designed for steering the initial state $\left|\psi_{0}\right\rangle$ into the target state $\left|\psi_{\text {target }}\right\rangle$ which is reachable from the eigenstate $\left|\phi_{g}\right\rangle$. 


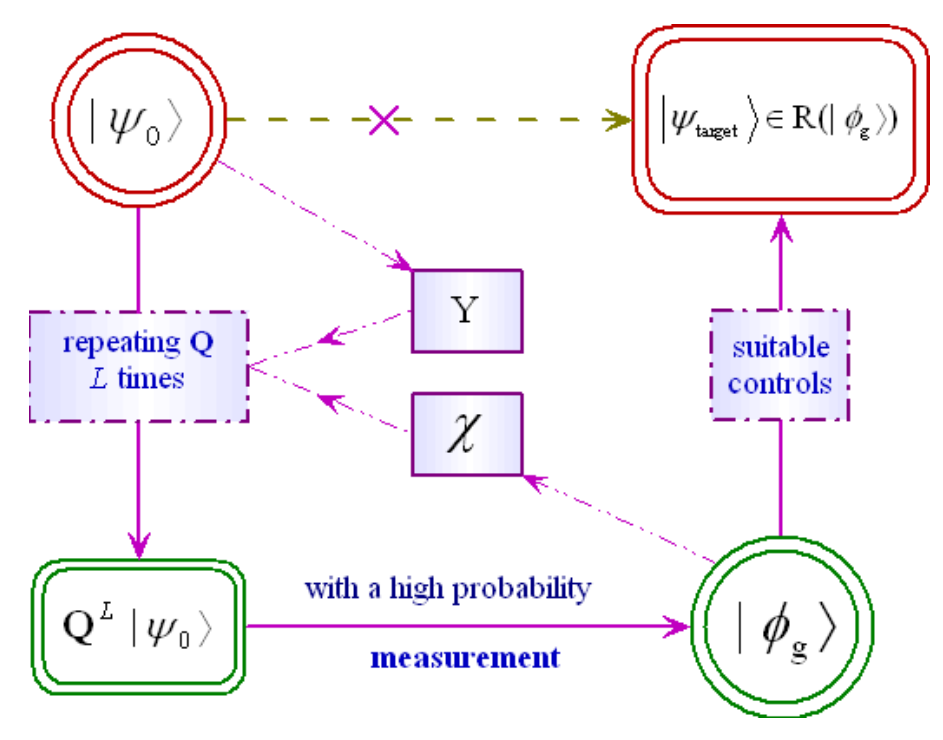

FIG. 1: (Color online) The schematic description of Algorithm 1.

\section{Algorithm 1 Incoherent Control Algorithm for Eigenstate Reachable Target States}

(1) Determine a unitary operator $\mathcal{U}$ such that $\left|\psi_{0}\right\rangle=\mathcal{U}\left|\phi_{1}\right\rangle$, and define $\left|\phi_{g}\right\rangle$ as the good state (i.e., corresponding to $\chi=1$ ) and the other eigenstates as bad states (i.e., corresponding to $\chi=0)$;

(2) Select a pair $\left(\varphi_{1}, \varphi_{2}\right)$ and construct the operation $\mathbf{Q}\left(\mathcal{U}, \chi, \varphi_{1}, \varphi_{2}\right)$;

(3) Find an optimal number of iterations $L$ and enhance the amplitude of $\left|\phi_{g}\right\rangle$ by applying the operation $\mathbf{Q}\left(\mathcal{U}, \chi, \varphi_{1}, \varphi_{2}\right)$ to the system L times;

(4) Make a measurement on the system, which will induce, with high probability, collapse of the system wavefunction into the state $\left|\phi_{g}\right\rangle$;

(5) Select a suitable control to drive $\left|\phi_{g}\right\rangle$ into $\left|\psi_{\text {target }}\right\rangle$.

Now we consider a special class of locally controllable quantum systems which have a wavefunction controllable subspace $\Omega$. $\Omega$ is taken as a good subspace, i.e., the eigenstates in $\Omega$ correspond to $\chi=1$, and we assume that the target state $\left|\psi_{\text {target }}\right\rangle$ is reachable from the eigenstates in $\Omega$. Without loss of generality, we assume $D_{\omega}=\left\{\left|\phi_{1}\right\rangle, \ldots,\left|\phi_{M}\right\rangle\right\}(1<M<N)$ and decompose $\left|\psi_{0}\right\rangle$ into two parts:

$$
\left|\psi_{0}\right\rangle=\sum_{i=1}^{M} c_{0 i}\left|\phi_{i}\right\rangle+\sum_{i=M+1}^{N} c_{0 i}\left|\phi_{i}\right\rangle=\left|\Phi_{g}\right\rangle+\left|\Phi_{b}\right\rangle,
$$

where $\left|\Phi_{g}\right\rangle=\sum_{i=1}^{M} c_{0 i}\left|\phi_{i}\right\rangle$ and $\left|\Phi_{b}\right\rangle=\sum_{i=M+1}^{N} c_{0 i}\left|\phi_{i}\right\rangle$. Then, an operator $\mathbf{Q}\left(\mathcal{U}, \chi, \varphi_{1}, \varphi_{2}\right)$ is constructed to enhance the amplitude of $\left|\Phi_{g}\right\rangle$. In analogy with the Algorithm 1, below we 


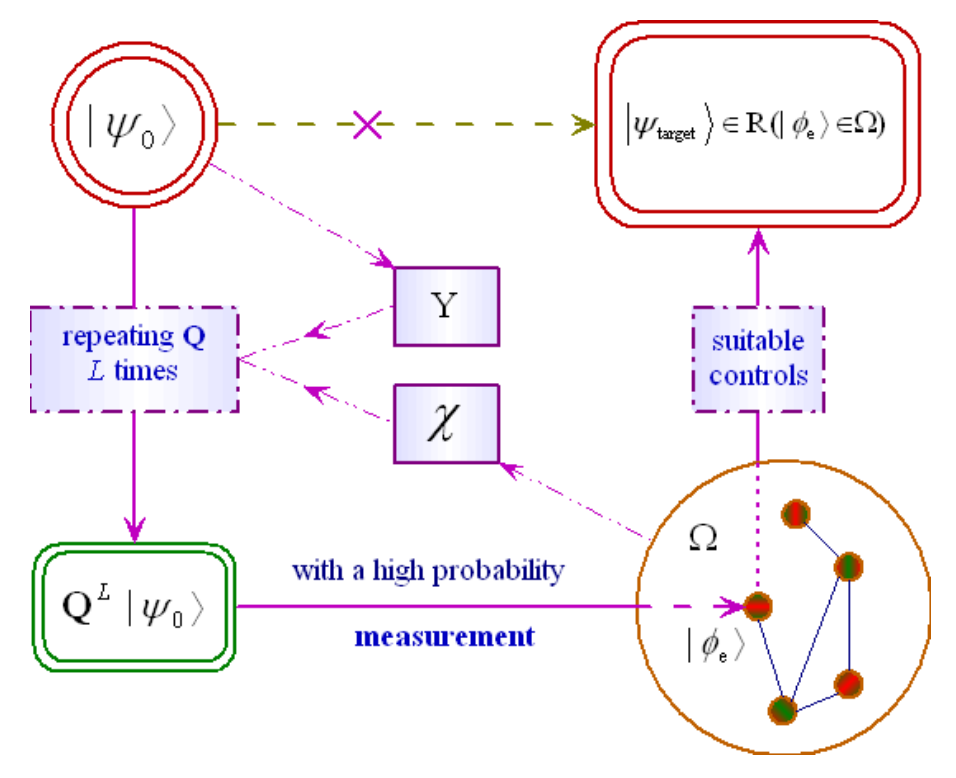

FIG. 2: (Color online) The schematic description of Algorithm 2.

describe the control algorithm Algorithm 2 for steering the initial state $\left|\psi_{0}\right\rangle$ of a quantum system, with a wavefunction controllable subspace $\Omega$, into the target state $\left|\psi_{\text {target }}\right\rangle$ which is reachable from the eigenstates in $\Omega$. A schematic of the algorithm is shown in Fig. 2 ,

Algorithm 2 Incoherent Control Algorithm for Quantum Systems with Wavefunction Controllable Subspaces

(1) Take $\Omega$ as the good subspace such that the eigenstates in $\Omega$ correspond to $\chi=1$ and the other eigenstates correspond to $\chi=0$;

(2) Decompose the initial state $\left|\psi_{0}\right\rangle$ as the sum $\left|\psi_{0}\right\rangle=\left|\Phi_{g}\right\rangle+\left|\Phi_{b}\right\rangle$ of the projection $\left|\Phi_{g}\right\rangle$ onto the good subspace and the projection $\left|\Phi_{b}\right\rangle$ onto the bad subspace;

(3) Determine $\mathcal{U}$ such that $\left|\psi_{0}\right\rangle=\mathcal{U}\left|\phi_{1}\right\rangle$, select a pair $\left(\varphi_{1}, \varphi_{2}\right)$ and construct $\mathrm{Q}\left(\mathcal{U}, \chi, \varphi_{1}, \varphi_{2}\right)$

(4) Find an optimal number of iterations $L$ and enhance the amplitude of $\left|\Phi_{g}\right\rangle$ by applying the operation $\mathbf{Q}\left(\mathcal{U}, \chi, \varphi_{1}, \varphi_{2}\right)$ to the system $L$ times;

(5) Make a measurement on the amplified state, which will induce collapse of the system wavefunction into a state $\left|\phi_{e}\right\rangle \in \Omega$ with high probability;

(6) Find a local optimal control sequence in $\Omega$ and a set of suitable controls to drive $\left|\phi_{e}\right\rangle$ into $\left|\psi_{\text {target }}\right\rangle$. 
Remark 1 In the proposed control algorithms, $g=\left\langle\Phi_{g} \mid \Phi_{g}\right\rangle$ cannot be equal to 0 . If $g=0$ for the initial state, we can make it non-zero by applying a suitable unitary transformation to the initial state $\left|\psi_{0}\right\rangle$ before using quantum amplitude amplification. For globally controllable systems the amplitude amplification step is not necessary, and the maximum probability of success is naturally equal to 1. The selection of an optimal $\mathbf{Q}$ for the two algorithms above remains an open issue. In Algorithm 2, after we project the conditional state into an eigenstate of the wavefunction controllable subspace, we can find a local optimal control sequence by quantum reinforcement learning [51], or we can design different controllers for every eigenstate ahead of time and complete the control task by a variable structure control method.

Remark 2 In the present incoherent control scheme, the measurement is used as a control tool and the expected selectivity is enhanced at the cost of overall conversion efficiency as the same as the case in other measurement-assisted incoherent control schemes. For this class of quantum systems where we know some information about local controllability, in this paper we use quantum amplitude amplification technology to enhance the expected selectivity and reduce the population loss in the measurement process.

\section{AN EXAMPLE: HYDROGEN ATOM}

Consider the hydrogen atom with the internal Hamiltonian

$$
H_{0}=-\frac{\hbar^{2}}{2 m} \Delta-\frac{e^{2}}{r}
$$

The wavefunction of the ground state in spherical coordinates $(r, \theta, \varphi)$ is

$$
\left|\psi_{100}(r, \theta, \varphi)\right\rangle=\frac{1}{\sqrt{\pi a^{3}}} e^{-r / a}
$$

where $a=0.53 \times 10^{-10} \mathrm{~m}$ is the Bohr radius. The first excited state is four-fold degenerate with the four corresponding wavefunctions

$$
\begin{aligned}
\left|\psi_{200}(r, \theta, \varphi)\right\rangle & =\frac{1}{\sqrt{8 \pi a^{3}}}\left(1-\frac{r}{2 a}\right) e^{-r / 2 a}, \\
\left|\psi_{210}(r, \theta, \varphi)\right\rangle & =\frac{1}{4 \sqrt{2 \pi a^{3}}} \frac{r}{a} e^{-r / 2 a} \cos \theta \\
\left|\psi_{211}(r, \theta, \varphi)\right\rangle & =-\frac{1}{8 \sqrt{\pi a^{3}}} \frac{r}{a} e^{-r / 2 a} \sin \theta e^{\iota \varphi}, \\
\left|\psi_{21-1}(r, \theta, \varphi)\right\rangle & =\frac{1}{8 \sqrt{\pi a^{3}}} \frac{r}{a} e^{-r / 2 a} \sin \theta e^{-\iota \varphi} .
\end{aligned}
$$


We restrict consideration to these first five eigenstates and denote them as $\left\{\left|\phi_{a}\right\rangle,\left|\phi_{b 1}\right\rangle,\left|\phi_{b 2}\right\rangle,\left|\phi_{b 3}\right\rangle,\left|\phi_{b 4}\right\rangle\right\}$ in the order listed above. In this model an arbitrary initial state $\left|\psi_{0}\right\rangle$ can be written as a superposition

$$
\left|\psi_{0}\right\rangle=c_{a}\left|\phi_{a}\right\rangle+\sum_{j=1}^{4} c_{b j}\left|\phi_{b j}\right\rangle
$$

Now consider the control design of this system. The natural simple way to control the state transitions is by using a time-dependent electric field $E_{z}(t)$ along the $z$-axis. The corresponding control Hamiltonian $H_{u}$ has the form

$$
H_{u}=-e E_{z}(t) z
$$

The wavefunction at time $t$ evolving under the action of this control Hamiltonian can be expressed as

$$
|\psi(t)\rangle=c_{a}(t)\left|\phi_{a}\right\rangle e^{-\iota E_{a} t / \hbar}+\left[\sum_{i=1}^{4} c_{b i}(t)\left|\phi_{b i}\right\rangle\right] e^{-\iota E_{b} t / \hbar},
$$

where $E_{a}$ and $E_{b}$ denote the energies of the ground and the first excited state, respectively, and the coefficients $C(t)=\left\{c_{a}(t), c_{b 1}(t), c_{b 2}(t), c_{b 3}(t), c_{b 4}(t)\right\}$ evolve according to the equation

$$
\dot{C}(t)=T C(t)
$$

Here the $T$ matrix is [52]:

$$
T=\left(\begin{array}{ccccc}
0 & 0 & \frac{128 \sqrt{2} \iota}{243 \hbar} a e E_{z}(t) e^{-\iota\left(E_{b}-E_{a}\right) t / \hbar} & 0 & 0 \\
0 & 0 & -\frac{3 \iota}{\hbar} a e E_{z}(t) & 0 & 0 \\
\frac{128 \sqrt{2} \iota}{243 \hbar} a e E_{z}(t) e^{-\iota\left(E_{b}-E_{a}\right) t / \hbar} & -\frac{3 \iota}{\hbar} a e E_{z}(t) & 0 & 0 & 0 \\
0 & 0 & 0 & 0 & 0 \\
0 & 0 & 0 & 0 & 0
\end{array}\right) .
$$

It follows from the above matrix that

$$
c_{b 3}(t)=c_{b 3}(0), \quad c_{b 4}(t)=c_{b 4}(0) .
$$

Thus, the amplitudes of the states $\left|\phi_{b 3}\right\rangle$ and $\left|\phi_{b 4}\right\rangle$ remain constant under the control Hamiltonian $H_{u}$, i.e., they are non-controllable under unitary evolution. This circumstance is similar to the example of Eqs. (14) and (15) in [19]. Here the states $\left|\phi_{b 3}\right\rangle$ and $\left|\phi_{b 4}\right\rangle$ are not 


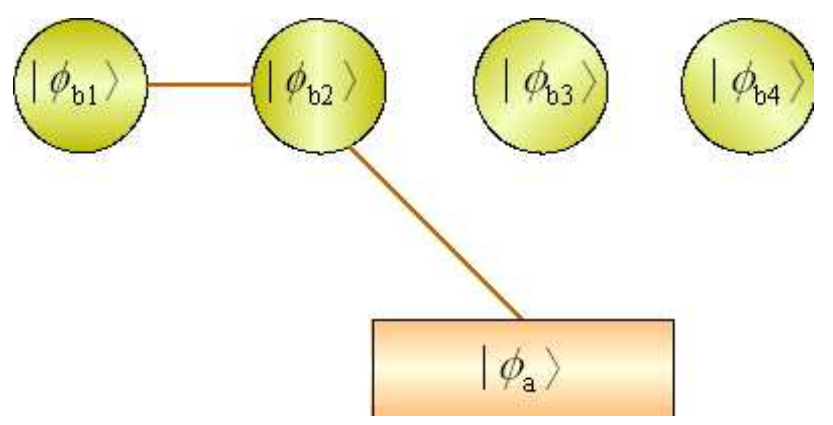

FIG. 3: (Color online) The state transitions of the hydrogen atom under the action of the control Hamiltonian $H_{u}$.

controllable because the field cannot effectively interact with those states due to the selected polarization. There are no necessary coupling interactions since the corresponding elements in the matrix $T$ are equal to zero. The state transitions are shown in Fig. 3. As mentioned in Sec. IIC, the degeneracy condition (I) in Theorem 2 can be relaxed in some circumstances. Although $\left|\phi_{b 1}\right\rangle$ and $\left|\phi_{b 2}\right\rangle$ are degenerate, the linear span of the set $D_{\omega}=\left\{\left|\phi_{a}\right\rangle,\left|\phi_{b 1}\right\rangle,\left|\phi_{b 2}\right\rangle\right\}$ forms a wavefunction controllable subspace $\Omega$, since the elements of $D_{\omega}$ are connected in Fig. 3 [52]. Now we consider the control design of the hydrogen atom for two special classes of target states. The limited controllability with the Hamiltonian $H_{u}$ will not be enough for performing these tasks and applying the electric field along the $x$-axis or $y$-axis, or both the $x$-axis and $y$-axis will be necessary.

Case 1: $\left|\psi_{\text {target }}\right\rangle \in R\left(\left|\phi_{b 4}\right\rangle\right)$

Here we consider the situation when the target state $\left|\psi_{\text {target }}\right\rangle$ is a state reachable from $\left|\phi_{b 4}\right\rangle$. It can be for example an excited state above the four-degenerate first excited states (i.e., a state with the principal quantum number $n=3,4,5, \ldots$ ). The assumption of the reachability of the target state assumes the existence of an electric control field which can transfer the state $\left|\phi_{b 4}\right\rangle$ into the target state.

Consider the initial state

$$
\left|\psi_{0}\right\rangle=0.7\left|\phi_{a}\right\rangle+0.5\left|\phi_{b 1}\right\rangle+0.3\left|\phi_{b 2}\right\rangle+0.4\left|\phi_{b 3}\right\rangle+0.1\left|\phi_{b 4}\right\rangle
$$

The amplitudes of the different eigenstates are schematically shown in the left subplot of Fig. 4. The numbers $\{1,2,3,4,5\}$ on the horizontal axis correspond to the states $\left\{\left|\phi_{a}\right\rangle,\left|\phi_{b 1}\right\rangle,\left|\phi_{b 2}\right\rangle,\left|\phi_{b 3}\right\rangle,\left|\phi_{b 4}\right\rangle\right\}$. The values along the vertical axis are the corresponding amplitudes. If we make a measurement of the projector $P_{b 4}=\left|\phi_{b 4}\right\rangle\left\langle\phi_{b 4}\right|$ on the initial state, 

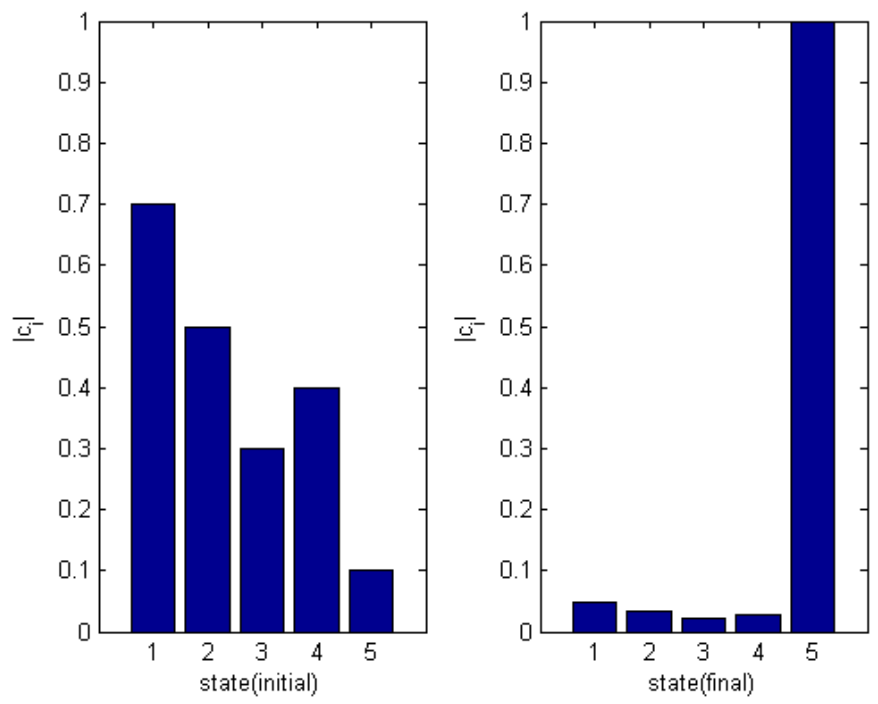

FIG. 4: The amplitudes of the eigenstates of the initial (left) and final (right) states for the Case 1. The integers along the $\mathrm{x}$-axis label the hydrogen atom states.

the probability of success is only $\left|c_{b 4}(0)\right|^{2}=1 \%$, and it is too small. Now use Algorithm 1 to enhance the probability of success. For convenience, we only use the special quantum amplitude amplification operation $\mathbf{Q}(\mathcal{U}, \chi, \pi, \pi)$. Generation of such operator $\mathbf{Q}(\mathcal{U}, \chi, \pi, \pi)$ will require applying the electric field along the $x$-axis or $y$-axis, or both the $x$-axis and $y$-axis. Using Theorem 4, we can compute the state of the system after repeated action of $\mathbf{Q}(\mathcal{U}, \chi, \pi, \pi)$ to the initial state seven times. The resulting amplitudes of the final state are shown on the right subplot of Fig. 4. Then we make a measurement of the projector $P_{b 4}$ on the final state. After the measurement the system will collapse into the state $\left|\phi_{b 4}\right\rangle$ with a high probability of $99.53 \%$. Then the last step of the incoherent control scheme is implemented by applying the coherent unitary control steering the state $\left|\phi_{b 4}\right\rangle$ into the target state $\left|\psi_{\text {target }}\right\rangle$.

Case 2: $\left|\psi_{\text {target }}\right\rangle \in R\left(\left|\phi_{e}\right\rangle \in \Omega\right)$

Consider the initial state

$$
\left|\psi_{0}\right\rangle=0.1\left|\phi_{a}\right\rangle+0.06\left|\phi_{b 1}\right\rangle+0.08\left|\phi_{b 2}\right\rangle+0.7\left|\phi_{b 3}\right\rangle+0.7\left|\phi_{b 4}\right\rangle
$$

The amplitudes of the different eigenstates are schematically shown on the left subplot of Fig. 5. If we measure the projector $P_{\Omega}$ onto the subspace $\Omega$, the probability of success for the initial state is only $\left|c_{a}(0)\right|^{2}+\left|c_{b 1}(0)\right|^{2}+\left|c_{b 2}(0)\right|^{2}=2 \%$. We now use Algorithm 2 to 

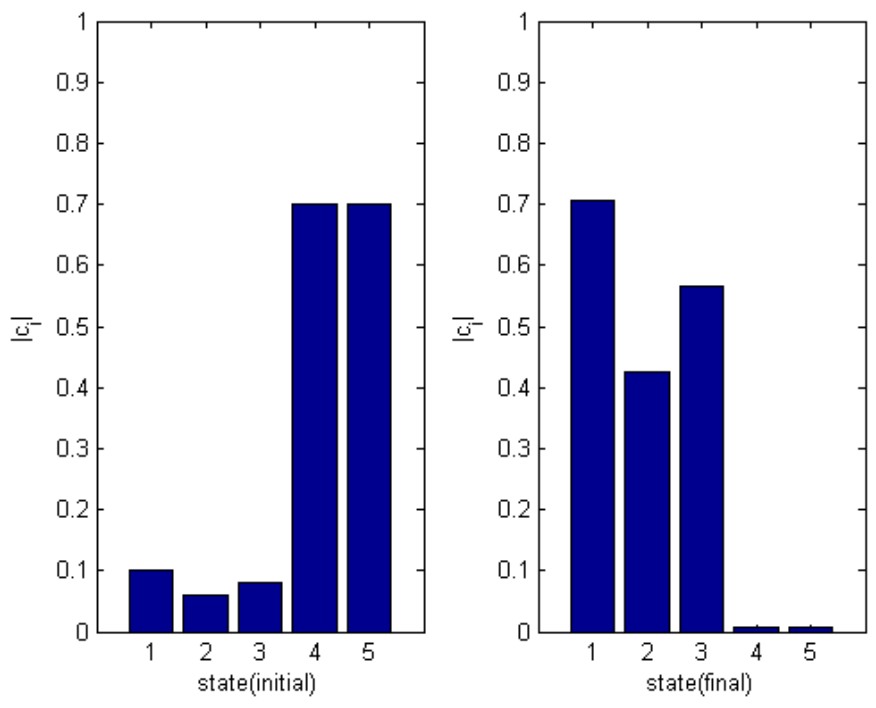

FIG. 5: The amplitudes of the eigenstates of the initial (left) and final (right) states for the Case 2.

enhance the probability of success by applying the operation $\mathbf{Q}(\mathcal{U}, \chi, \pi, \pi)$ to the system five times. Theorem 4 allows for computing the resulting amplitudes, which are shown on the right subplot of Fig. 5. Then we make a measurement on the amplified state, and the state will collapse into subspace $\Omega$ with a high probability $99.99 \%$. After projecting the final state into the subspace $\Omega$, we can use learning control or variable structure control to drive the state into the target state $\left|\psi_{\text {target }}\right\rangle$.

Remark 3 To demonstrate the present incoherent control scheme, we gave a simple example of a hydrogen atom controlled by an external field. For two special cases, we designed the corresponding amplitude amplification operator $\mathbf{Q}(\mathcal{U}, \chi, \pi, \pi)$ (a unitary transformation). The unitary transformation can be constructed using the method presented in Section III.B according to nature of the initial states and local controllability information. One goal of this work is to establish a connection between control design and controllability analysis, and also demonstrate that local controllability information can be used to design control laws for quantum systems. Further, it is necessary to generate effective external control field to realize the known unitary transformation $\mathbf{Q}(\mathcal{U}, \chi, \pi, \pi)$. In this example, the control field should include some components along some other axes (e.g., $x$-axis, $y$-axis, or both the x-axis and y-axis). The details of control field design are left to future work. Moreover, our control scheme can easily be applied to multiple level systems such as the five level system in [53]. If 
we know some information about local controllability and there are fewer nonzero component values in the electric dipole transition matrix than those given in [53] and [19], we can use a method similar to the one used in this example to design the control law.

\section{CONCLUDING REMARKS}

Many of the works studying the controllability of quantum systems are devoted to the analysis of global properties of controlled quantum systems. However, some quantum systems may not be globally controllable or sometimes information about global controllability may be difficult to deduce, and we can then consider operation with locally controllable quantum systems. In fact, a system with a controllable subspace is a special case of locally controllable systems. Systems with controllable subspaces have practical importance in quantum information technology [29], e.g., systems with a DFS in quantum computation [27]. It is valuable to steer the system into a DFS and to maintain the encoded information in the DFS for the computation operations through suitable control schemes [29, 54]. We believe that the present incoherent control scheme can provide an alternative method for such problems.

In the present control scheme, quantum measurement is used as an effective control to probabilistically project the amplified state into a desired eigenstate. This scheme uses controlled unitary transformations supplemented by quantum measurements and thus can be considered within the class of measurement-assisted schemes [39, 41]. Since the quantum measurement makes the state collapse probabilistically, our incoherent control scheme is also a probabilistic control strategy, similar in some sense to the incoherent control scheme of [49]. However, the methods of enhancing the probability of success are quite different. In [49], the probability of success is enhanced by multiple measurements on identical initial states. Generally it requires several identical initial states but they may be unknown. In the present scheme, quantum amplitude amplification is used to increase the probability of success, and the initial state should be known. In this sense, the present scheme is similar

to the quantum control scheme based on the use of Grover iterations [55]. However, the quantum control algorithm in [55] relies on the assumption that the system is eigenstate controllable, which may be difficult to verify. Moreover, the construction of Grover iterations also has some special requirements, which may not be suitable for control design 
of general quantum systems. The present scheme overcomes these drawbacks and is more suitable for accomplishing practical control tasks. Here we only require the system to be locally controllable and use the general amplitude amplification technique to increase the

probability of success. The amplitude amplification operations can be directly realized by the construction of a suitable control Hamiltonian. It is obvious that this incoherent control scheme is also suitable for the control of globally controllable quantum systems.

In conclusion, we propose an incoherent control scheme for a class of quantum systems exhibiting local controllability. In this scheme, we only need to know partial controllability information about the system. The paper also provides an example with the hydrogen atom to demonstrate the operation of the scheme. In our method, quantum measurements are used as an effective control and the gap between a controllability analysis and control design is bridged. The experimental details for implementing the scheme need to be explored for the practical manipulation of quantum systems.

\section{Acknowledgments}

The authors would like to thank the reviewer of J. Chem. Phys. for helpful comments which have greatly improved this paper. D.D. would like to thank Prof. Lei Guo and Dr. Bo Qi for helpful suggestions. This work was supported in part by the National Natural Science Foundation of China under Grant No.60703083, the National Creative Research Groups Science Foundation of China under Grant No.60421002 and the China Postdoctoral Science Foundation (20060400515). H. Rabitz and A. Pechen thank DOE for support. A. Pechen also thanks RFFI 08-01-00727-a for a partial support. 
[1] H. Rabitz, R. de Vivie-Riedle, M. Motzkus and K. Kompa, Science, 288, 824 (2000).

[2] Z. H. Chen, D. Y. Dong and C. B. Zhang, Quantum Control Theory: An Introduction (Hefei: University of Science and Technology of China Press, 2005) (in Chinese).

[3] H. Rabitz, Theor. Chem. Acc., 109, 64 (2003).

[4] M. Shapiro and P. Brumer, Principles of the Quantum Control of Molecular Processes (New Jersey: John Wiley \& Sons, Inc., 2003).

[5] M. Dantus and V. V. Lozovoy, Chemical Reviews, 104, 1813 (2004).

[6] R. S. Judson and H. Rabitz, Phys. Rev. Lett., 68, 1500 (1992).

[7] S. Chu, Nature, 416, 206 (2002).

[8] V. Bonacic-Koutecky and R. Mitric, Chemical Reviews, 105, 11 (2005).

[9] M. A. Nielsen and I. L. Chuang, Quantum Computation and Quantum Information (Cambridge, England: Cambridge University Press, 2000).

[10] M. Grace, C. Brif, H. Rabitz, I. A. Walmsley, R. L. Kosut and D. A. Lidar, J. Phys. B: At. Mol. Opt. Phys. 40, S103 (2007).

[11] M. Grace, C. Brif, H. Rabitz, D. A. Lidar, I. A. Walmsley and R. L. Kosut, J. Modern Optics 54, 2339 (2007).

[12] J. P. Dowling and G. J. Milburn, Phil. Trans. R. Soc. Lond A, 361, 1655 (2003).

[13] G. M. Huang, T. J. Tarn and J. W. Clark, J. Math. Phys., 24, 2608 (1983).

[14] V. Ramakrishna and H. Rabitz, Phys. Rev. A, 54, 1715 (1996).

[15] V. Ramakrishna, M. V. Salapaka, M. Dahleh, H. Rabitz and A. Peirce, Phys. Rev. A, 51, 960 (1995).

[16] R. B. Wu, T. J. Tarn and C. W. Li, Phys. Rev. A, 73, 012719 (2006).

[17] F. Albertini and D. D'Alessandro, IEEE Trans. Autom. Control, 48, 1399 (2003).

[18] S. G. Schirmer, H. Fu and A. I. Solomon, Phys. Rev. A, 63, 063410 (2001).

[19] G. Turinici and H. Rabitz, Chem. Phys., 267, 1 (2001).

[20] G. Turinici and H. Rabitz, J. Phys. A: Math. Gen., 36, 2565 (2003).

[21] C. Altafini, J Math. Phys., 44, 2357 (2003).

[22] R. Wu, A. Pechen, C. Brif and H. Rabitz, J. Phys. A: Math. Theor., 40, 5681 (2007).

[23] K. Beauchard, J. Math. Pures Appl., 84, 851 (2005). 
[24] K. Beauchard and J. M. Coron, J. Funct. Anal., 232, 328 (2006).

[25] A. Y. Khapalov, SIAM J. Control Optim., 46, 655 (2007).

[26] G. Farkas, J. Math. Chem., 24, 1 (1998).

[27] D. A. Lidar, I. L. Chuang and K. B. Whaley, Phys. Rev. Lett., 81, 2594 (1998).

[28] P. G. Kwiat, A. J. Berglund, J. B. Altepeter and A. G. White, Science, 290, 498 (2000).

[29] M. Kiffner, J. Evers and C. H. Keitel, Phys. Rev. A, 75, 032313 (2007).

[30] S. Lloyd, Phys. Rev. A, 62, 022108 (2000).

[31] M. Shapiro and P. Brumer, J. Chem. Phys., 84, 4103 (1986).

[32] W. S. Warren, H. Rabitz and M. Dahleh, Science, 259, 1581 (1993).

[33] S. A. Rice, Nature, 409, 422 (2001).

[34] S. A. Rice and M. S. Zhao, Optical Control of Molecular Dynamics (New York: John Wiley \& Sons, Inc., 2000).

[35] R. Vilela Mendes and V. I. Man'ko, Phys. Rev. A, 67, 053404 (2003).

[36] A. Mandilara and J. W. Clark, Phys. Rev. A, 71, 013406 (2005).

[37] J. B. Gong and S. A. Rice, J. Chem. Phys., 120, 9984 (2004).

[38] M. Sugawara, J. Chem. Phys., 123, 204115 (2005).

[39] A. Pechen, N. Il'in, F. Shuang and H. Rabitz, Phys. Rev. A, 74, 052102 (2006).

[40] L. Roa, A. Delgado, M. L. Ladrón de Guevara and A. B. Klimov, Phys. Rev. A, 73, 012322 (2006).

[41] F. Shuang, A. Pechen, T. S. Ho and H. Rabitz, J. Chem. Phys., 126, 134303 (2007).

[42] A. Pechen and H. Rabitz, Phys. Rev. A, 73, 062102 (2006).

[43] A. Pechen and H. Rabitz, Preprint http://arxiv.org/abs/0801.3467.

[44] R. Romano and D. D’Alessandro, Phys. Rev. Lett., 97, 080402 (2006).

[45] R. Romano and D. D'Alessandro, Phys. Rev. A, 73, 022323 (2006).

[46] G. Brassard, P. Høyer and A. Tapp, Lecture Notes Comput. Sci., 1443, 820 (1998).

[47] P. Høyer, Phys. Rev. A, 62, 052304 (2000).

[48] L. K. Grover, Phys. Rev. Lett., 80, 4329 (1998).

[49] D. Dong, C. Chen, T. J. Tarn, A. Pechen and H. Rabitz, IEEE Trans. Syst., Man, Cybern., Part B: Cybern. 38, 957 (2008).

[50] G. Brassard and P. Høyer, in Proc. of the 5th Israeli Symposium on Theor. Comput. Syst., $12(1997)$. 
[51] D. Dong, C. Chen, H. Li and T. J. Tarn, Quantum reinforcement learning, IEEE Trans. Syst., Man, Cybern., Part B: Cybern. 38, 1207 (2008).

[52] C. B. Zhang, Research on Controllability and Control Strategy of Quantum systems under No-Unitary Evolution (Ph.D. thesis, University of Science and Technology of China, 2007) (in Chinese).

[53] S. H. Tersigni, P. Gaspard and S. A. Rice, J. Chem. Phys., 93, 1670 (1990).

[54] P. Cappellaro, J. S. Hodges, T. F. Havel, D. G. Cory, J. Chem. Phys., 125, 044514 (2006).

[55] C. B. Zhang, D. Y. Dong and Z. H. Chen, J. Opt. B: Quantum Semiclass. Opt., 7, S313 $(2005)$. 